\title{
Impact of AI on Environment
}

\author{
Dr.Indradeep Verma ${ }^{a}$ \\ ${ }^{a}$ Associate Professor, CSE Department, IIMT College of Engineering Greater Noida, Uttar Pradesh, India. \\ Mr. Gambhir Singh ${ }^{b}$ \\ ${ }^{b}$ Associate Professor, CSE Department, IIMT College of Engineering Greater Noida, Uttar Pradesh, India.
}

\author{
Abhuday Tiwari ${ }^{\mathrm{c}}$ \\ ${ }^{c}$ UG Students, CSE Department, IIMT College of Engineering Greater Noida, Uttar Pradesh, India.
}

Amit Kumar

${ }^{d}$ UG Students, CSE Department, IIMT College of Engineering Greater Noida,Uttar Pradesh, India.

Harish Prasad ${ }^{\mathrm{e}}$

,e UG Students, CSE Department, IIMT College of Engineering Greater Noida,Uttar Pradesh, India.

Article History: Received: 10 November 2020; Revised 12 January 2021 Accepted: 27 January 2021;

Published online: 5 April 2021

\begin{abstract}
$\underline{\text { Abstract }}$
Nowadays we see and use various AI(artificial intelligence) solutions in our daily life for solving a myriad of day-to-day problems. Various fields are being found and researched on where artificial intelligence can be used for making systems more efficient but do we know about the cost our environment pays for our use of these solutions implemented using AI and machine learning. The computational cost of the AI solutions should be considered too. We introduce you to these costs incurred in the environment and some solutions to minimize them.
\end{abstract}

Keywords:- AI, Environment, Machine Learning, Computational Cost, Artificial Intelligence

\section{Introduction}

AI is claimed as the replication of human perception within the machine which is designed to make perceptions like man and may replicate their gestures. This can also be used on a machine which can exhibit human like characteristics such as problem solving and learning new things. Artificial Intelligence has various applications in today's society, many industries such as healthcare, education, finance, entertainment have implemented AI for solving different complex problems. As it can solve various complex problems efficiently it has made our daily life easy and comfortable. 
But while we use artificial intelligence to solve these problems, have we ever considered what cost must the environment pay for implementation of such AI based solutions. There are many areas and fields related to artificial intelligence that we are expanding our research to but have we thought about the carbon footprint it leaves. One might not think that it could cost much to our environment but have we ever tallied the costs. In order to achieve accuracy in the machine learning models, they are trained repeatedly without much consideration of the computational costs as there are resources available right now.

\section{How much does it cost?}

For quantification of computational cost, a life cycle assessment of many AI models was performed at researchers at Massachusetts. This computational cost in terms of $\mathrm{CO} 2$ emissions came out to be five times the average lifetime $\mathrm{CO} 2$ emissions of an American car.

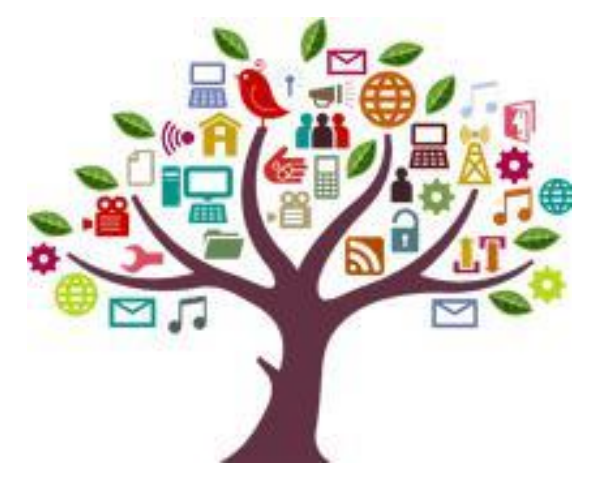

\section{AI Applications in Environmental Sector}

Several MNCs and small scale companies, are trying to cross the boundaries for human alterations, are making large attempts in evolving 'Eco Friendly' Artificial Intelligence machines. For Example, Google's own DeepMind AI assisted the company to restrain their data center energy consumption by $40 \%$ increasing their energy efficiency and decreasing overall GHG discharge. Development of such kind of AI is necessary to improve energy efficiency because their data centers consume $3 \%$ of global energy every year. Development of such kind of system also helps in facilitation of energy access to distinct communities, building up integrating renewable energy resources and microgrids.

By installing microgrids we can utilize artificial intelligence techniques in cities to command and manage power grids in neighbourhood to supply the exact amount of power supply required, or demanded from the households, where in conventional systems power is wasted due to unorganized distribution of power supply.

\section{Procedures}

To measure the cost of environmental and computing price of training deep neural network models for Natural Language Processing, we carry out the evaluation of the amount of energy required to train a grow of well-liked off the shelf Natural Language Processing models, also a 
detailed study of the whole sum of assets essential to make LISA, a state of the art Natural Language Processing model from Empirical Methods in Natural Language Processing 2018, consisting of all the tuning and examinations.

\section{$\underline{\text { 2.a Designs }}$}

We examine 4 designs, the computing needs of which we outline beneath. Codes of each and every model is available online for free, which we have used out of the box. Please refer to the original papers for detailed information of the designs.

Transformer:- This model is primarily recognised for exact and precise machine translations. It is an encoder-decoder architecture. 6 stacked layers of multi-head self attention is consisted in each encoder and decoder. On 8 NVIDIA P100 GPU's the transformer base model (65M parameters) was coached for 12 hours and for 84 hours the transformer big model (213M parameters) in 300,000 steps. For Natural Language Processing pipeline, modelling of language and Neural Architecture Search the transformer model is used as a basis. So, the base model needs 10 hours to train for 300,000 steps on one TPU core and complete architecture search run for 979,000,000 steps. This means 11421.66 days on 8 P100 GPU's or 1359.291 days on TPUv2.

ELMo:- This model lays out a effective way to characterize words in context by pre-training using language modelling targets on huge data sets. In downstream tasks such as conference, entity identification and participant role labelling using ELMo in place of subject-independent pre-coached word embeddings has shown an increase in the performance. On 3 NVIDIA GTX 1080 GPUs ELMo was coached for 14 days.

BERT:- This model is used for designing subjective representations like ELMo model but instructed under a different language modelling objective. This model gives a transformer based architecture. In tasks where sentence level representation is required like answering question and inferencing of natural language BERT greatly enhances efficiency. On 16 TPU chips BERT base model (110M parameters) takes 4 days to be trained. NVIDIA claims that using 4 DGX-2H servers, which totals around $2^{6}$ Tesla V100 GPUs BERT model can be trained in 3.3 days.

GPT-2 :- it is based on transformer style self attention and instructed with a language modelling purpose. It is the newest version of OpenAI's GPT general purpose token encoder. Ranford et al.(2019) displays giant zero shot execution on language modelling basis and answering questions. This large model needs 7 days instructing on 32 TPUv3 chips and has 15,420,00,000 parameters.

\begin{tabular}{ccccc} 
Customers & Natural Gas Coal Energy & Nuclear & $\begin{array}{c}\text { Renewable } \\
\text { Resources }\end{array}$ \\
\hline \hline United States & $7 / 20$ & $27 / 100$ & $19 / 100$ & $17 / 100$ \\
\hline \hline China & $3 / 100$ & $13 / 20$ & $1 / 25$ & $11 / 50$ \\
\hline \hline Germany & $7 / 100$ & $19 / 50$ & $13 / 100$ & $2 / 5$ \\
\hline \hline Microsoft & $23 / 100$ & $31 / 100$ & $1 / 10$ & $8 / 25$ \\
\hline \hline Amazon & $6 / 25$ & $3 / 10$ & $13 / 50$ & $17 / 100$ \\
\hline \hline Google & $7 / 50$ & $3 / 20$ & $1 / 10$ & $14 / 25$
\end{tabular}


Table 1: Fraction values of energy obtained from: Renewable Resources (e.g. hydro, solar, wind), natural gas, coal energy and nuclear energy for the highest three cloud evaluate suppliers, contrast to the US, 4 China 5 and Germany.

\section{Experimental results}

\section{3.a Cost of training}

\begin{tabular}{ccccccc}
\hline Design & Machinery & Days & Power(W) & CO2e Cloud Evaluation & kWh-PUE \\
Cost & & \\
\hline NAS & TPUv2x1 & 1359.291 & ---- & ---- & $€ 36563-€ 121875$ & ---- \\
\hline ELMo & P100x3 & 14 & 517.66 & 26 & $€ 359-€ 1221$ & 275 \\
\hline $\begin{array}{c}\text { Transformer } \\
\text { Base Model }\end{array}$ & P100x8 & 0.5 & 1415.78 & 26 & $€ 34-€ 116$ & 27 \\
\hline GPT-2 & TPUv3x32 & 7 & ---- & ---- & $€ 10707-€ 35694$ & ---- \\
\hline $\begin{array}{c}\text { BERT Base } \\
\text { Model }\end{array}$ & TPUv2x16 & 4 & ---- & ---- & $€ 1721-€ 5736$ & --- \\
\hline $\begin{array}{c}\text { Transformer } \\
\text { Top Model }\end{array}$ & P100x8 & 3.5 & 1515.43 & 192 & $€ 239-€ 814$ & 201 \\
\hline $\begin{array}{c}\text { BERT Base } \\
\text { Model }\end{array}$ & V100x64 & 3.291 & 12041.51 & 143.8 & $€ 3113-€ 10433$ & 1507 \\
\hline NAS & P100x8 & 11421.66 & 1515.43 & 626,155 & $€ 782611-$ & 656,347 \\
\hline
\end{tabular}

Table 2: List of approximate cost of instructing designs as discussed in 2.a and CO2 release. Important thing is that GPUs are more costlier than TPUs on the loads of work that recognize the given hardware(e.g.BERT). It is also seen that models discharge considerable amount of carbon release i.e. coaching BERT model on GPU is approximately equal to the trans-American flight. For English to German machine translation NAS attains a new state of the art BLEU score of 29.7, and for at the minimum cost of $€ 125488.89$ an increase of just 0.1 BLEU on request evaluation time and non-trivial carbon release.

\section{$\underline{\text { 3.b }}$ Building Cost: Detailed study}

To evaluate the computing needs for Research and Development of a new design(model) we go through all the logs of training needs to make Linguistically Well-versed self-attention, a multitasking model which can perform part-of-speech tagging, parsing of the labelled dependencies, labelling of semantic roles and detection of predicates. As an illustrative of best long paper at EMNLP and NLP pipeline, this model develops a fascinating case study. Training of model related to project covered a period of approximately 6 months. At that point of time 123 little hyperparameter grid searches were carried out, leading to a total of 4789 jobs. Jobs diverse long starting from at least 3 minutes, signifying a crash, to an upper limit of 9 days, with 52 hours as mean job length. All the coaching was completed on a combination of $28 \%$ of M40 GPUs and $72 \%$ of NVIDIA TITAN X. The total GPU time needed by the project sum to 239,952 hours. This means to about 60 GPUs running constantly throughout the extent of project. Table 3 lists the lower and upper bounds of the approximate cost in reference to Google cloud evaluation and 
power required by this model to deploy and develop. It is seen that training one model is relatively inexpensive, the worth of tuning a model for a replacement dataset, which is predicted to need about 24 jobs, carrying out the full research and development needed to make this model , spontaneously became tremendously costly.

\section{Approximate cost (Euros)}

\begin{tabular}{cccc}
\hline Models & Days & Cloud Evaluation Cost & Power \\
\hline 1 & 5 & $€ 42.92-€ 144.43$ & $€ 4.13$ \\
24 & 120 & $€ 1021.71-€ 3470.36$ & $€ 97.38$ \\
4789 & $9,997.583$ & $€ 85.01 \mathrm{k}-€ 288.85 \mathrm{k}$ & $€ 8145.65$ \\
\hline
\end{tabular}

Table 3: Approximate cost of cloud evaluation and power for instructing: (1)a single model (2)a single tune (3) all models instructed during R\&D.

\section{Conclusion}

With deteriorating environment we would not want AI and machine learning to add more to it. Thus, we must find solutions for minimizing the computational cost of these models. Further measures that can be adopted to do so can be as follows:-

- Think before you train, we must always think first before training a model if the training of the model will achieve desired results, training models repeatedly to achieve accuracy by a very small measure may incur very high costs thus we must always consider the feasibility keeping in mind the computational cost.

- Better optimizing of datasets, we must find ways for better optimising of datasets which could further decrease the computational cost of the model.

- Tallying the costs, we must calculate the costs associated with the models for better determination if the feasibility of the model training.

- Promotion of usage of alternative and renewable resources, the more renewable resources are used instead of the non-renewable resources the more we can bring down the harmful impact of AI and ML computational costs on the environment.

- Tiny AI

- The next advancement in AI and ML is tiny AI, more research should be promoted on tiny AI as this solution is made to dramatically reduce the size of the algorithms which can be built on small hardware or on devices with low power consumption thus ultimately reducing the threat to nature.

\section{References:}

[1]. https://en.m.wikipedia.org/wiki/Artificial_intelligence

[2]. https://www.forbes.com/sites/forbestechcouncil/2020/08/17/why-we-should-care-about-th eenvironmental-impact-of-ai/amp/

[3]. Artificial Intelligence Tutorial - Tutorialspoint 
[4]. Stuart Russell, Peter Norvig, "Artificial Intelligence - A Modern Approach”, Pearson Education

[5]. Elaine Rich and Kevin Knight, "Artificial Intelligence", McGraw-Hill

[6]. E Charniak and D McDermott, "Introduction to Artificial Intelligence", Pearson Education

[7]. Dan W. Patterson, "Artificial Intelligence and Expert Systems", Prentice Hall of India

[8]. Tom M. Mitchell, —Machine Learning, McGraw-Hill Education (India) Private Limited, 2013

[9]. Ethem Alpaydin, - Introduction to Machine Learning (Adaptive Computation and Machine Learning), The MIT Press 2004

[10]. Stephen Marsland, - Machine Learning: An Algorithmic Perspective, CRC Press, 2009

[11]. Bishop, C., Pattern Recognition and Machine Learning. Berlin: Springer-Verlag

[12]. https://www.climate-kic.org/opinion/ai-and-climate-change-the-promise-the-perils-and-pillars-foraction/\#: :text=First $\% 2 \mathrm{C} \% 20 \mathrm{AI} \% 20$ systems $\% 20$ have $\% 20$ the,both $\% 20 \mathrm{impact} \% 20$ and $\% 20$ resource $\% 20 \mathrm{dec}$ oupling.

[13]. https://xd.adobe.com/ideas/principles/emerging-technology/ai-impact-on-climate-environment/ 